\title{
Secondary Data Issues and Non-generalizability of Findings
}

\author{
Elysia Desgrosseilliers
}

Published online: 5 February 2014

(C) Springer Science+Business Media New York 2014

To the Editor,

In the article, "The Role of Individual and Neighborhood Factors: HIV Acquisition Risk Among High-Risk Populations in San Francisco," Raymond et al. examine socioeconomic status and social and sexual network factors and their relationship to HIV acquisition risk among HIVnegative white MSM (WMSM), black MSM (BMSM) and transfemales (male to female transgenders). There are two hypotheses stated in the article: (1) "that a neighborhood factor such as population HIV prevalence shape sexual network composition and may have a stronger relationship to HIV acquisition among HIV uninfected persons than individual SES or individual behavior." [1] and (2) "that persons using stimulants are more likely to engage in HIV acquisition risk and reside in neighborhoods with high HIV prevalence and have high HIV prevalence in their sexual networks." [1] While there is value in exploring these hypotheses, Raymond et al. do not make a conclusion on the second hypothesis and stimulant use is not addressed in their discussion.

Raymond et al. identified three cohorts: WMSM, BMSM and transfemales. While the sample populations are large, the data for each cohort come from different data sources and from different years with some limitations to the generalizability of their findings. The data for WMSM were taken from the National HIV Behavioral surveillance System (NHBS) collected in 2008. The NHBS was not limited to WMSM, but the authors only used the data of WMSM from this source. The data for BMSM were taken from an HIV testing program in 2009 run by the HIV Prevention Section of the San Francisco Department of

E. Desgrosseilliers $(\square)$

Memorial University of Newfoundland, St. John's, NL, Canada

e-mail: elysiad@mun.ca
Public Health. The intention of this program was to deliver information to injecting drug users; therefore, this sample population of BMSM would have presumably higher injecting drug use than the actual BMSM population. The data for transfemales were collected from the $2010 \mathrm{HIV}$ Prevention Section risk assessment: transfemales empowered to advance community health. Participants may not reflect the general transfemale population seeing as this risk assessment would have attracted participants with an interest in community health. This potentially led to a sample population that takes less HIV risks than the rest of the transfemale population.

While this study brought about new information regarding socioeconomic status and social and sexual network factors and their relationship to HIV acquisition risk, the discussion was at times unclear and did not address the second stated hypothesis. Additionally, issues with the secondary data sources take away from the generalizability of their findings and limit their conclusions to their sample populations. Nevertheless, Raymond et al. justly conclude that interventions will need to address multiple factors to reduce HIV risk taking and this study may be used as a basis for future research addressing neighborhood factors on HIV acquisition risk.

\section{Reference}

1. Raymond HF, Chen Y-H, Syme SL, Catalano R, Hutson MA, McFarland W. The role of individual and neighborhood factors: HIV acquisition risk among high-risk populations in San Francisco. AIDS Behav. 2013;18(2):346-56. doi:10.1007/s10461-0130508-y. 